\title{
Palmitic acid induces inflammation in hypothalamic neurons via ceramide synthesis
}

\author{
D. Sergi, D.E. Kahn, A.C. Morris and L.M. Williams \\ Rowett Institute of Nutrition and Health, University of Aberdeen, Greenburn Road, Aberdeen AB21 9SB
}

The consumption of a high-fat diet, especially those rich in long chain saturated fatty acids, has been reported to induce hypothalamic inflammation and reactive gliosis in rodents ${ }^{(1,2)}$. Increased hypothalamic gliosis has also been found in obese humans ${ }^{(2)}$. The hypothalamus is pivotal in the regulation of energy balance but this breaks down in obesity. Hypothalamic inflammation induced by the consumption of long chain saturated fatty acids arises prior to the onset of obesity indicating that it may be causative in the development of obesity ${ }^{(2)}$, particularly, as it promotes central insulin and leptin resistance ${ }^{(3,4)}$. In contrast, monounsaturated and polyunsaturated fatty acids have been reported to protect against obesity and related metabolic dysfunction ${ }^{(5)}$.

In light of this, we investigated whether palmitic acid (PA), a long chain saturated fatty acid, and a major component of the Western diet was able to elicit an inflammatory response in hypothalamic neurons and whether the n-3 polyunsaturated fatty acid, eicosapentaenoic acid (EPA) and the monounsaturated fatty acid, oleic acid (OA) could ameliorate PA induced inflammation. To do this N42 (mHypoE-N42) neurons were treated with $200 \mu \mathrm{M}$ PA in the presence or absence of $125 \mu \mathrm{M}$ OA or EPA. Statistical analysis was carried out using Student's t-test. PA upregulated the expression of both $T N F \alpha$ and $I L-6$ as assessed by real-time PCR while the addition of EPA or OA alongside PA significantly abrogated $T N F \alpha$ and $I L-6$ upregulation by PA alone.

As a high-fat diet has been shown to promote ceramide accumulation in the hypothalamus ${ }^{(6)}$ and ceramide induces lipotoxicity and endoplasmic reticulum stress ${ }^{(7)}$, we evaluated whether PA induced upregulation of pro-inflammatory cytokine genes was dependent on ceramide accumulation. N42 neurons were treated with PA in the presence or absence of L-cycloserine, an inhibitor of ceramide synthesis. PA was found to increase cellular C16 ceramide content relative to control cells and L-cycloserine blocked the PA induced ceramide accumulation as quantified by LC MS. L-cycloserine also significantly downregulated the expression of PA induced $I L-6$ and $T N F \alpha$. To ascertain whether EPA and OA exert their anti-inflammatory effects via a decrease in cellular ceramide levels, we also measured C16 ceramide content in neurons treated with $200 \mu \mathrm{M}$ PA in the presence and absence of EPA or OA. Both EPA and OA successfully decreased the PA induced increase in ceramide content.

In summary, our results show that PA induces an increase in C16 ceramide and an inflammatory response in cultured N42 hypothalamic neurons both of which are inhibited by EPA, OA and L-cycloserine. Thus, ceramide accumulation represents one on the mechanisms by which PA induces inflammation and whereby EPA and OA may block that induction.

1. De Souza CT, Araujo EP, Bordin S, et al. (2005) Endocrinology 146, 4192-4199.

2. Thaler JP, Yi CX, Schur EA, et al. (2012) J Clin Invest 122, 153-162.

3. Zhang X, Zhang G, Zhang H, et al. (2008) Cell 135, 61-73.

4. Purkayastha S, Zhang G, Cai D (2011) Nat Med 17, 883-887.

5. Cintra DE, Ropelle ER, Moraes JC, et al. (2012) PLoS One 7, e30571.

6. Borg ML, Omran SF, Weir J, et al. (2012) J Physiol 590, 4377-4389.

7. Contreras C, Gonzalez-Garcia I, Martinez-Sanchez N, et al. (2014) Cell Rep 9, 366-377. 